\title{
Dependability Modelling and Evaluation of Cyber-Physical Systems: A Model- Driven Perspective
}

\author{
Hu Xiaojing, Liu Shixi, Chen Guilin \\ Computer Science and Technology Department \\ Chuzhou University \\ Anhui Chuzhou, China \\ liusxchuz@163.com
}

\begin{abstract}
The strong coupling of software and physical processes in the emerging field motivates the development of new methods to respond to dependability in both the cyber and physical domains. Two kinds of definitions of CyberPhysical System (CPS) from the view of resource, service and process is presented. The dependable Model of CyberPhysical Home Control System is presented by taking advantage of Stochastic Petri Net (SPN). Based on the model running and analyzing, the dependency relationships in resource, service and process, and the dependability parameters (Reliability, Availability, Sustainability, MTTF and etc.) of system which people pay attention to are presented. If this model and method are applicable to CPS, that means the formal methods and verification based on model-driven are appropriate to CPS.
\end{abstract}

Keywords- Dependability, Model-driven, cyber-physical system, Stochastic Petri Nets

\section{INTRODUCTION}

Cyber-physical system is a system featuring a tight combination of and coordination between the system's computational and physical elements. Today, a pre-cursor generation of cyber-physical systems can be found in areas as diverse as aerospace, automotive, chemical processes, civil infrastructure, energy, healthcare, manufacturing, transportation, entertainment, and consumer appliances. Given the enormous social and economic value of the CPS applications, the CPS design and development methods research has become the new hot spot of common concern in today's international industry and academia ${ }^{[1,2]}$.

Unlike more traditional embedded systems, a fullfledged CPS is typically designed as a network of interacting elements with physical input and output instead of as standalone devices ${ }^{[2]}$.CPS is often used in safety critical areas with high reliability and predictability. However, how to build a highly reliable and predictable model of the CPS is a challenging task.

The difficulty of modeling language to build CPS lies in the diversity of the system characteristics for CPS. CPS is running in an open environment, so the physical device status interacting with the computing system is not completely predictable, and the system operating environment is not fully under control. Even if the physical device error or abnormal operating environment occurs, the CPS must have the robustness and fault tolerance to accomplish tasks properly.

The CPS system consists of discrete components and continuous components. Not only is it necessary to achieve the functional requirements, but also to meet nonfunctional requirements. It shows behavioral

\author{
Jiang Cuiqing \\ School of Management \\ Hefei University of Industry \\ Anhui Hefei, China \\ jiangcuiq@163.com
}

characteristics of the coexistence of both synchronous and asynchronous, determinism and randomness. However, the traditional design methods can not support the design of complex model of the CPS effectively. A new method is necessary for analyzing, testing, verifying CPS model components and integrated systems at different levels with a variety of temporal characteristics and spatial characteristics.

Since the cyber-physical systems are composed of software interacting with the physical world, many classes of faults exist. On the cyber side, there are timing failures of real-time programs and operating systems, in addition to crash failures, and simply software bugs. On the physical side, there are actuator, control surface, and sensor failures, aside from of course necessary robustness given the potential operating environments of a system. Between these two worlds is the potential for communication failures, such as message drops and omissions, or worse, adversarial man-in-the-middle attacks perhaps culminating in Byzantine failures ${ }^{[3]}$.

When the delivered service of a CPS deviates from fulfilling the system intended function, and then the CPS has a failure. A failure is due to a deviation from the correct state of the CPS, known as error. Such a deviation is due to a given cause, for instance related to the physical state of the system, or to a bad system design. This cause is called a fault. It generically refers to fault, errors, and failures as the "FEF elements" "[4]. Any of these FEF elements can result in a degradation of physical state, and thus potential violation of safety or liveness, which in the context of safety-critical systems must be handled appropriately to prevent catastrophic failures.

The dependability means the ability of system to provide service continuously and renew all of the service in the certain time, when it is affected by exterior attack, error operation, environment impact or software bugs which cause failure occurs ${ }^{[5]}$. In the evaluation of a computing system, the dependability is a very important indicator system. The formal description is taken to express the extent of dependability for computing system. The dependability includes many parameter indices, such as reliability, survivability, availability, mean time to failure $(\boldsymbol{M T T F})$, meantime to repair $(\boldsymbol{M T T R})$, mean time between faults $(\boldsymbol{M T B F})$ and etc..

In this paper, the dependability modelling and analysis of Cyber-Physical Systems is taken into account from a model-driven perspective.

The remainder of this paper is arranged as follows: Section II introduces the related work; Section III introduces typical architecture of Cyber-Physical Home Control System, and presents two kinds of definitions of CPS from the view of resource, service and process. 
Based on moden-driven technology, the SPN model of Cyber-Physical Home Control System architecture is presented, and the dependency between faults, errors, failure is analyzed; In Section IV the dependability indicators of CPS is introduced and computed based on the SPN model; The last section summarizes the conclusion and claims the future work.

\section{RELATED WORK}

Professor Edward Lee presents the challenges of the design of CPS. He pointed out that the key requirements of CPS are reliability and predictability. To achieve the potential of the CPS system, constructing an abstract model of the new computing and networking is needed. The model should combine the physical dynamics and computing elements properly ${ }^{[7]}$.

A failure logic for each component allows for automated analysis of the system. Here, the failure logic depends only on the inputs, which is appropriate for modeling software failures, but cannot explicitly use failure states of the component itself.

Taylor Johnson points that potential directions in Cyber-Physical Systems are the handling of failures ${ }^{[3]}$. The complexity of this problem, solely in the classes of faults, is great. In addition to exploring the applicability of the existing literature and the new proposed methods to CPS, people must also utilize first principles such as abstraction to deal with this complexity, as otherwise that will wind up over designing these new systems. Some interesting questions are, given a system and a model, when is it possible to detect failures; Can time be bounded to detection; how do we minimize time to detection; Can we utilize physical state to more easily or quickly identify failures.

Linda Briesemeister presents an implementation of a probabilistic logic model, which allows for reasoning via symbolic evaluation as well as numeric evaluation to perform a quantitative fault analysis ${ }^{[8]}$. The models are built from a few building blocks, which can be instantiated as standard or high integrity; communication paths can be made redundant, and finally, whole subsystem blocks can be replicated.

The new requirements of system modeling are put forward for the complexity of the structure, behavior and data of CPS [9]. "Abstract technology" is an important method which people used to understand complex systems, and the formal methods provide mathematical foundation for abstraction technique. The formal models based on state machine are fit to describe the structure and behavior of system. The typical methods include Statecharts ${ }^{[10,11]}$ and Petri nets ${ }^{[12,13]}$.

The formal model based on the temporal logic is applied to describe the properties of system ${ }^{[14]}$. There are also many research results in the formal model for hybrid systems with discrete computational components and continuous variables ${ }^{[15]}$. Fusion of two or more complementary formal methods in modeling complex systems is also quite common ${ }^{[16]}$.The main problem to use the two formal methods at the same time is how to integrate them coordinately and meaningfully.

Formal methods and verification will provide useful tools for solving these problems. Sha motivated in ${ }^{[17]}$ the need for formal methods in detecting and mitigating faults in what are now termed CPS, and more recently again in as there are countless further directions to explore $^{[18]}$. While thus far they have primarily considered mitigating faults to avoid violation of safety properties, they propose also a study of optimizing liveness properties in addition to maintaining safety.

The most applicable methods in the controls literature to CPS are probably model-based techniques. Given that effectively all CPS must maintain some notion of the current states of the system with regards to time to be able to interact with the physical world, the real-time systems community has analyzed faults.

\section{THE SPN MODEL OF THE CYBER-PHYSICAL SYSTEM}

\section{A. The Definition of Cyber-physical system}

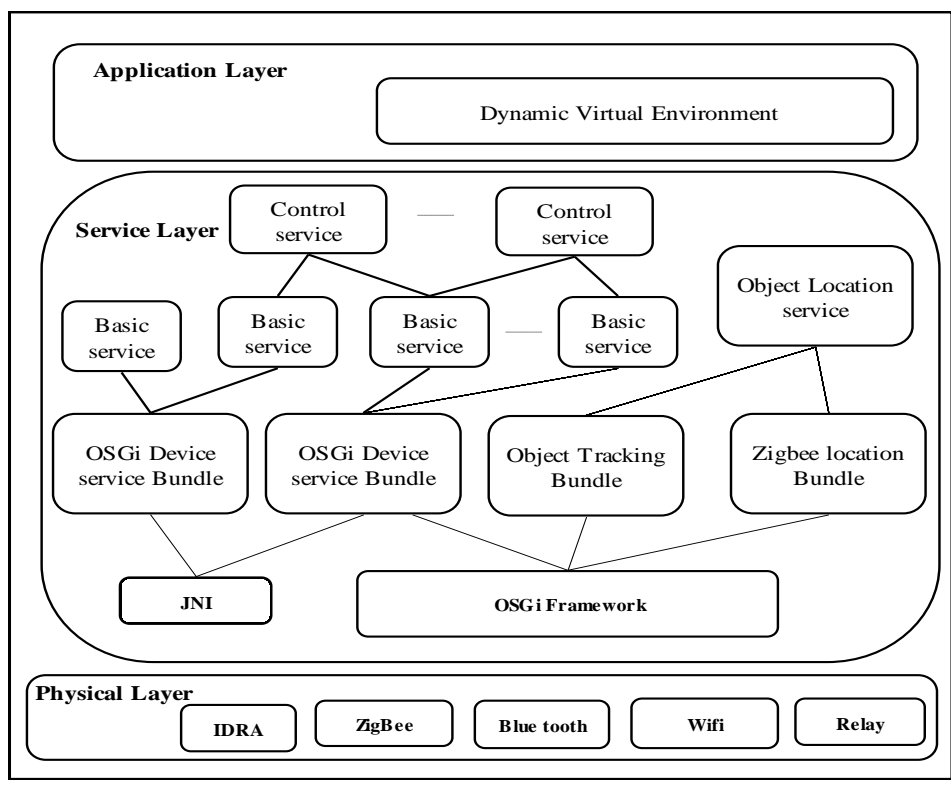

Fig. 1. Cyber-Physical Home Control System architecture

Fig. 1 shows the entire architecture of the CyberPhysical Home Control System ${ }^{[19]}$. The Cyber-Physical Home Control System proposed contains 3 layers: the Physical Layer, the protocols and profiles for users control of home appliances; the Service Layer, the conversion and management of physical layer signals and application layer services, through device drivers in the OSGi Bundle, control signals are in packet formats, which conform to the specific protocols of the home appliances of the Physical Layer; the Application Layer, all the services provided by physical equipment can be converted to the OSGi service model for users, through the Service Layer. Details of the various elements of the system architecture proposed are described as in reference [19].

This section presents extensions that allow fault, error, and failure elements to be modeled together in a CPS architectural style. A formalized definition of Cyberphysical system free of faults, errors, failures is presented by Definition 1.

Definition $1 C P S=\{R, S, P\}, R=\left\{\mathrm{r}_{1}, \mathrm{r}_{2}, \ldots, \mathrm{r}_{\mathrm{m}}\right\} ; S=\left\{\mathrm{s}_{1}\right.$, $\left.\mathrm{s}_{2}, \ldots, \mathrm{s}_{\mathrm{n}}\right\} ; P=\left\{\mathrm{p}_{1}, \mathrm{p}_{2}, \ldots, \mathrm{p}_{\mathrm{t}}\right\}$.

The CPS is organized into three levels: resources, 
services and processes represented by R, S, P respectively. Resources are at the bottom level, and they provide operations for the services, where a service is basically a complex pattern of use of the resources. Services are then requested by the application model placed at the highest level, called process level.

A formalized definition of Cyber-physical system with faults, errors, failures is presented by Definition 2 .

Definition $2 C P S=\{R, S, P\}, R=\left\{R^{\prime}, F T\right\}, S=\left\{S^{\prime}, E\right\}$, $P=\left\{P^{\prime}, F n\right\} ; R^{\prime}=\left\{\mathrm{r}_{1}, \mathrm{r}_{2}, \ldots, \mathrm{r}_{\mathrm{m}}\right\}, F T=\left\{\mathrm{ft}_{1}, \mathrm{ft}_{2}, \ldots, \mathrm{ft}_{\mathrm{m}},\right\}$; $S^{\prime}=\left\{\mathrm{s}_{1}, \mathrm{~s}_{2}, \ldots, \mathrm{s}_{\mathrm{n}}\right\}, E=\left\{\mathrm{e}_{1}, \mathrm{e}_{2}, \ldots, \mathrm{e}_{\mathrm{n}}\right\} ; P^{\prime}=\left\{\mathrm{p}_{1}, \mathrm{p}_{2}, \ldots, \mathrm{p}_{\mathrm{t}}\right\}$, $F n=\left\{\mathrm{fn}_{1}, \mathrm{fn}_{2}, \ldots, \mathrm{fn}_{\mathrm{t}},\right\}$.

It assumes that there are a number of models for faults, errors, and failures, called $F T_{-j}, E_{-h}$, and $F_{-n}$, respectively, and it provides a schematic view of how they are organized in three levels: fault models are placed at the resource level, error models at the service level and failure models at process level. we assume that faults affect only the behaviour of the resources of the CPS, errors are perceived at the service level, while failure are a concern of CPS, and therefore of the process level.

\section{B. Intoduction to Stochastic Petri nets}

Stochastic Petri nets are graphical and mathematical modelling tools applicable to many systems. They are promising tools for describing and studying information processing systems that are characterized as being concurrent, asynchronous, distributed, parallel, nondeterministic ${ }^{[12]}$. SPN model expresses a complex system in the form of graph, and its structure elements include Place, Transition and Arc. There are two kinds of methods for Arc to regulate relation between local states and events: (1) They quote local states in which events can occur; (2) The local states' conversion that is caused by events.

Definition $3^{[12]}$ Stochastic Petri Net is a quadruple $(P$, $T ; F, \lambda$ ) where

(1) $P$ is a finite set of places;

(2) $T$ is a finite set of transitions $(P \cap T \neq \varphi)$;

(3) $F \subseteq(P \times T) \cup(T \times P)$ is a set of arcs;

(4) $\lambda=\left(\lambda_{1}, \lambda_{2}, \ldots, \lambda_{n}\right)$, is a set of firing rates of transitions set.

The state space of SPN will increase exponentially along with problems becoming more complex. However, it makes the isomorphic Markov Chain of SPN hard to solve. The presentation of GSPN(Generalized Stochastic Petri net) offers an effective approach to solve the problem of state space explosion. The GSPN is the extension of SPN, and the representation is as follows: The transitions are divided into two categories, one is transient transition that is associated with stochastic switch and its firing time is zero; the other is time transition that is associated with exponential distributed firing time. The GSPN model is processed to obtain the associated dependability measures (i.e., availability, reliability, Survivability, and etc.) using an evaluation tool such as Stochastic Petri Net Package(SPNP), deterministic and stochastic Petri nets(DSPN), and etc..

\section{The SPN model of Resource Service and Process for CPS}

Figure 2 is the SPN model of Cyber-Physical Home Control System which is organized into three levels: resources, services and processes ${ }^{[20]}$. Resources are at the bottom level, and they provide operations for the services, where a service is basically a complex pattern of use of the resources. Services are then requested by the application model placed at the highest level, called process level. The top part of Figure 2 depicts a skeleton of the process model that uses services. Suppose process of CPS carried out by resource components and service components. Then, $\mathrm{P}_{\text {up }}$ and $\mathrm{P}_{\text {failure }}$ places' markings which mean the up state and down state of process depend upon the markings of the resource and service components models. The activation rate of a resource fault is $\lambda_{h}\left(T_{r 1}\right)$, and of a service error is $\lambda_{\mathrm{s}}\left(\mathrm{T}_{\mathrm{r} 6}\right) ; \mathrm{R}_{\mathrm{fa}}$ and $\mathrm{S}_{\mathrm{ea}}$ means fault activated and error activated respectively. The probability that a resource fault is temporary is $t\left(\mathrm{t}_{\mathrm{r} 1}\right)$. Such faults will disappear with rate $\varepsilon\left(\mathrm{T}_{\mathrm{r} 2}\right)$; A permanent resource fault (resp. service) is detected by the fault-tolerance mechanisms with probability $d_{h}$ (resp. $d_{s}$ for service faults). The detection rate is $\delta_{\mathrm{h}}\left(\mathrm{T}_{\mathrm{r} 3}\right)$ for the resource, and $\delta_{\mathrm{s}}\left(\mathrm{T}_{\mathrm{r} 7}\right)$ for the service; place $\mathrm{R}_{\text {fault }}$ and $\mathrm{S}_{\text {error }}$ means the resource and service of CPS is in fault state and error state respectively. The effects of a non detected error are perceived with rate $\pi_{R}\left(T_{r 4}\right)$ for the resource, and rate $\pi_{S}$ $\left(T_{r 8}\right)$ for the service; Place $R_{\text {fnd }}$ and $S_{\text {end }}$ means fault does not detected and so doesn't error repectively.

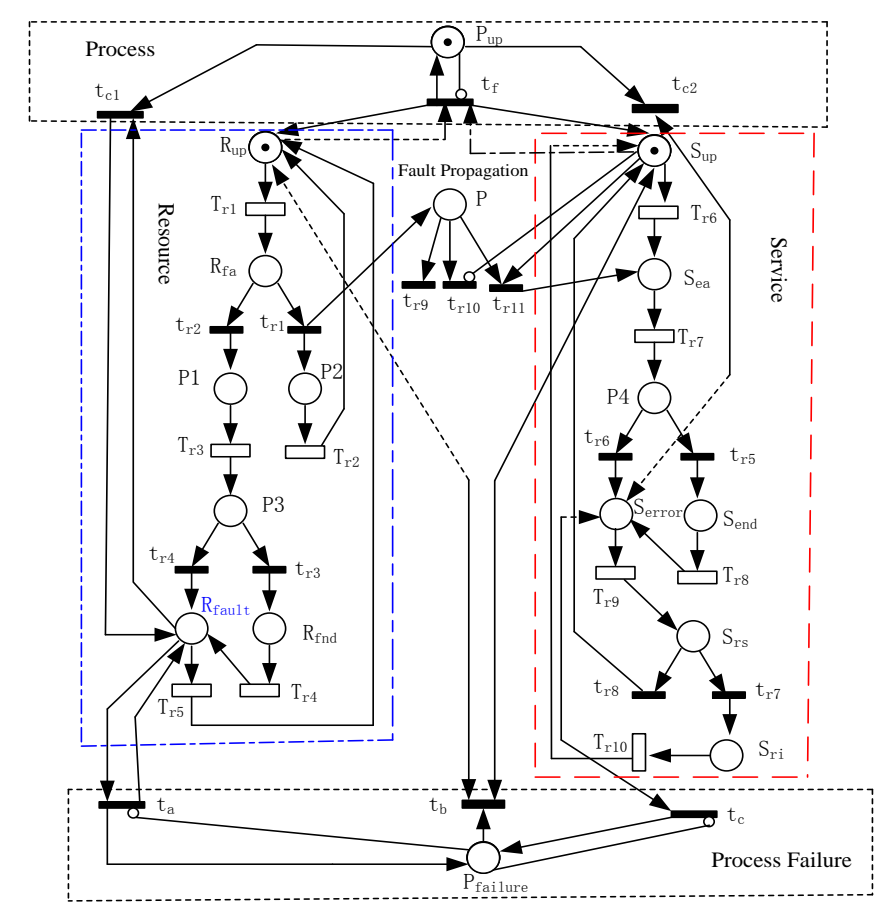

Fig . 2 The SPN model of Cyber-Physical Home Control System

Faults detected in the resource component require its repair: repair rate is $\mu\left(\mathrm{T}_{\mathrm{r} 5}\right)$; permanent errors in the service may necessitate only a reset, place $S_{\mathrm{rs}}$ means service reset. The reset rate is $\rho\left(\mathrm{T}_{\mathrm{r} 9}\right)$ and the probability that an error induced by the activation of a permanent service error disappears with a reset is $\gamma\left(\operatorname{tr}_{8}\right)$. If the error does not disappear with the service reset, a re-installation 
of the service is done, place $S_{\mathrm{ri}}$ means service reinstallation. The service's re-installation rate is $\sigma\left(\mathrm{T}_{\mathrm{r} 10}\right)$. In the following, dependency analysis between faults, errors, and failures is talked about.

Note that a temporary fault in the resource may propagate to the service $\left(\operatorname{tr}_{11}\right)$ with probability $\mathrm{p}$. When the service component is in place $S_{\text {error }}$, the service is in fact not available, i.e., in a error state(there is one token in $S_{\text {error }}$ place). It will provoke the firing of transition $t_{c}$, the token will move into place $P_{\text {failure }}$. That means the process of CPS fails because of the error of service. Also, when the resource is in the repair state, the service is on hold. The service will be reset or re-installed as soon as the resource repair is finished. In addition to the architectural dependencies, we take into account maintenance dependency between the two resources that share a repairman that is not simultaneously available for the two components. This maintenance dependency is not visible on the architectural models .

\section{DEPENDABILITY ANALYSIS OF CYBER-PHYSICAL} SYSTEM

\section{A. Introduction to dependability}

The term dependability is normally used to refer to the ability of an element (hardware or software component, plant or whatever complex system) to correctly perform its intended function, or mission, over time. We are interested in the quantitative evaluation of dependability of CPS. Since the failure of CPS will never be impossible, it is hoped that the system failure probability is low enough to meet their application requirements. It is believed that the behavior and results of dependable CPS can be predicted by solving the model. The introduction of dependability indicators in details is following ${ }^{[5]}$.

Reliability: means the continuity of Providing correct service, and it can be measured with $\mathrm{R}(\mathrm{t})$. It is generally believed that the probability of system works well in the time interval $(0, t]$ under the conditions of zero time. It is considered that the failure of system which has a Markov nature. The reliability can be expressed by $R(t)=p\{x>t\}$, and unreliability can be computed by $\mathbf{1}-\boldsymbol{R}(\boldsymbol{t})$.

Availability: means the capacity of system to provide services correctly. According to the relationship between availability and time, availability is divided into instantaneous availability, steady state availability, and intrinsic availability.

(1) Instantaneous availability $\mathbf{A}(\mathbf{t})$ : means the probability of system can provide services at any time after it begins to work.

(2) Steady state availability: means the expectations of the system which can be used to implement an effective service level.

$$
A_{s}=\lim _{t \rightarrow \infty} A(t)
$$

(3) Intrinsic availability: Only consider the service time, failure and repair time of equipment or system, regardless of the availability of other time. $A_{0}=M T B F /(M T B F+M T T R)$

Survivability: Refers to the capacity of system to complete its key services while the attacks, failures or accidents occur. It can be measured by the proportion of available resources after faults occur.

\section{B. Dependability analysis of CPS}

According to the dependability definition of CPS, we may compute different dependability indicators by solving the depenadability model. The steady state pobabilities of SPN model should be solved firstly. Given that there are $\mathrm{n}$ elements in the reachable marking set $\left[M_{0}\right\rangle$, namely there are $\mathrm{n}$ states in the Markov chain. Define $Q=\left[q_{i j}\right]$ as the state transition matrix. The state transition matrix $Q$ may be computed according to the following method ${ }^{[5]}$.

(1) when $\mathrm{i} \neq \mathrm{j}$, If $\mathrm{t}_{\mathrm{k}} \in \mathrm{T}: M_{i}\left[t_{k}>M_{j}\right.$ then

Else $q_{i, j}=0$;

$$
q_{i, j}=d\left(1-e^{-\lambda_{k} \tau}\right) /\left.d \tau\right|_{\tau=0}=\lambda_{k}
$$

(2) when $i=j$,

$$
q_{i, j}=d \prod_{k}\left(1-\left(1-e^{-\lambda_{k} \tau}\right)\right) /\left.d \tau\right|_{\tau=0}=d\left(e^{-\tau} \sum_{k} \lambda_{k}\right) /\left.d \tau\right|_{\tau=0}=-\sum_{k} \lambda_{k}
$$

Provided that there are $\mathrm{n}$ states of Markov Chain and its state probability is a row vector, namely $\left(p=p_{0}, p_{1}, \ldots\right.$, $\left.p_{\mathrm{n}}\right)$. According to Markov Stochastic process, the linear equation group is following:

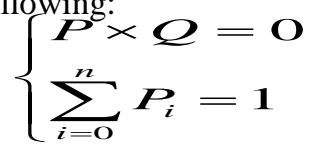

Here, we asume that $\lambda_{\mathrm{h}}=0.25, \lambda_{\mathrm{s}}=0.01, \varepsilon=0.23, \mathrm{~d}_{\mathrm{h}}=0.7$, $\mathrm{d}_{\mathrm{s}}=0.66, \delta_{\mathrm{h}}=0.25, \delta_{\mathrm{s}}=0.1, \pi_{\mathrm{R}}=0.24, \pi_{\mathrm{S}}=0.0 .26, \mu=0.05$ $\rho=0.02, \mathrm{p}=0.01, \gamma=0.8, \sigma=0.01$. We take advantage of SPNP Package developed by Duke University to compute the stable state probability of every reachable marking,

\begin{tabular}{ccc}
\hline State $_{\mathbf{i}}:$ Probability $_{\mathbf{i}}$ & State $_{\mathbf{i}}:$ Probability $_{\mathbf{i}}$ & State $_{\mathbf{i}}:$ Probability $_{\mathbf{i}}$ \\
\hline $0: 8.008008007514 \mathrm{e}-002$ & $1: 1.001001000763 \mathrm{e}-002$ & $2: 8.008008011030 \mathrm{e}-002$ \\
$3: 4.004004001140 \mathrm{e}-002$ & $4: 4.004004001140 \mathrm{e}-002$ & $5: 1.001001001381 \mathrm{e}-002$ \\
$6: 2.002002003169 \mathrm{e}-002$ & $7: 4.004004003019 \mathrm{e}-002$ & $8: 5.005005000144 \mathrm{e}-003$ \\
$9: 4.004004003019 \mathrm{e}-002$ & $10: 3.203203204907 \mathrm{e}-002$ & $11: 8.008008011180 \mathrm{e}-002$ \\
$12: 1.001001000845 \mathrm{e}-002$ & $13: 2.002002000820 \mathrm{e}-002$ & $14: 4.004004003045 \mathrm{e}-002$ \\
$15: 5.005005002703 \mathrm{e}-003$ & $16: 1.001001000845 \mathrm{e}-002$ & $17: 4.004004006834 \mathrm{e}-003$ \\
$18: 1.001001001570 \mathrm{e}-002$ & $19: 1.001001001487 \mathrm{e}-002$ & $20: 1.601601602152 \mathrm{e}-002$ \\
$21: 4.004004005578 \mathrm{e}-002$ & $22: 2.002002000628 \mathrm{e}-002$ & $23: 4.004004001932 \mathrm{e}-002$ \\
$24: 1.601601602152 \mathrm{e}-002$ & $25: 4.004004005578 \mathrm{e}-002$ & $26: 4.004004004225 \mathrm{e}-002$ \\
27:5.005005007632e-003 & $28: 5.005005001035 \mathrm{e}-003$ & $29: 1.001001000331 \mathrm{e}-002$ \\
$30: 2.002002002670 \mathrm{e}-003$ & $31: 5.005005007193 \mathrm{e}-003$ & $32: 5.005005007632 \mathrm{e}-003$ \\
$33: 5.005005005914 \mathrm{e}-003$ & $34: 2.002002002962 \mathrm{e}-002$ & $35: 8.008008005401 \mathrm{e}-003$ \\
$36: 2.002002001735 \mathrm{e}-002$ & $37: 1.601601600885 \mathrm{e}-002$ & $38: 4.004004002827 \mathrm{e}-002$ \\
$39: 2.002002002962 \mathrm{e}-002$ & $40: 2.502502502214 \mathrm{e}-003$ & $41: 5.005005003324 \mathrm{e}-003$ \\
$42: 2.502502504110 \mathrm{e}-003$ & $43: 1.001001001332 \mathrm{e}-002$ & $44: 2.002002002167 \mathrm{e}-002$ \\
\hline
\end{tabular}

namely, $p_{\mathrm{i}}\{\mathrm{t}=\infty\}=p_{\mathrm{i}}(0 \leq \mathrm{i} \leq 44)$ as follows.

table I. Stable State Probability of CPS

Based on stable state probability, we may analyze the dependable parameters of CPS which people may pay attention to.

\section{(1) Reliability}

When it comes to the dependability, whether it is the repairable system or not, we may suppose CPS has $n$ states. The first $t$ sequential normal working states are non-absorbing ones while the latter $a$ states are absorbing 
ones (failed states). Hence, one just needs to pay attention to the dependability of absorbed states. Namely,

$$
R(\mathrm{t})=p_{0}(t)=\exp \left(-\left(\lambda_{\mathrm{h}}+\lambda_{\mathrm{s}}\right) t\right)
$$

(2) Mean time to failure $(M T T F)$

If the failures of CPS elements obey to exponential distribution, then

$$
\text { MTTF }=\int_{0}^{\infty} R(t) d t=\int_{0}^{\infty} \exp \left(-\sum_{i=1}^{n} \lambda_{i} t\right) d t=\left(\lambda_{s}+\lambda_{h}\right)^{-1}
$$

(3) Mean time to repair and meantime between faults (MTTR, MTBF)

The average repairing time (average failure time)of system can be expressed by

$$
\operatorname{MTTR}=\sum_{i=0}^{n-1} \lambda_{i} / \sum_{i=0}^{n-1} \mu_{i}=\frac{\lambda_{s}}{\mu}+\frac{\lambda_{h}}{\sigma}
$$

The meantime between faults is closely equal to the ratio between the normal working time and the times of failure during this time, namely

(4) Availability

$$
M T B F=t / N_{f}(t)
$$

The stable availability of CPS is the stable probability of initial state $M_{0}$, then

\section{(5) Survivability}

$$
A_{s}=p_{0}=\left(1+\sum_{i=0}^{n-1} \lambda_{i} / \mu_{i}\right)^{-1}=\left(1+\frac{\lambda_{h}}{\mu}+\frac{\lambda_{s}}{\sigma}\right)^{-1}
$$

We can get the survivability of CPS which is expressed by $E[R]$, according to its definition.

$$
E[R]=\sum_{i=0}^{n-1} \frac{n-i}{n} p_{i}=\sum_{i=0}^{44} \frac{45-i}{45} p_{i}
$$

\section{CONCLUSION}

Dependability evaluation plays an important role in cyber-physical systems' definition, design and development. Dependability evaluation main objective is to assess the ability of a system to correctly function over time. There are many possible approaches to the evaluation of dependability of CPS. In these notes we are mainly concerned with dependability evaluation based on model-driven. In cyber-physical system, people are interested in characterization faults and how faults manifest as failures that affect overall reliability goals of the system. Formal methods and verification will provide useful tools for solving these problems. Stochastic Petri net is good at dynamically analyzing asychronism, concurrence and nondeterminism. In this paper, two kinds of definitions of cyber-physical system from the view of resource, service and process is presented. The dependability Model of Cyber-Physical Home Control System is presented by taking advantage of Stochastic Petri Net. Based on the model running and analyzing, the dependency relationships in resource, service and process, and the various dependability parameters of system which people pay attention to are presented with the help of SPNP. The approach based on model-driven has been exemplified with an example taken from the CPS domain.

\section{ACKNOWLEDGMENT}

The work presented in this paper is supported by the National Natural Science Foundation of China under Grant(No.61272153, No.61272153); Humanitiy and Social Science Foundation of Ministry of Education of China (13YJA630037); Natural Science Foundation of
Eduational government of Anhui Province (No.KJ2011ZD06, No.KJ2011B117, No.KJ2012Z282); Natural Science Foundation of ChuZhou university (No.2011kj008B, 2012qd09).

\section{REFERENCES}

[1] J. Sztipanovits, J. A. Stankovic, D. E. Corman (Eds). IndustryAcademy Collaboration in Cyber Physical Systems (CPS) Research White Paper. http://www.cra.org/ccc/docs/ CPS-White\%20Paper- May19-2009-GMU-v1.pdf, 2009.

[2] http://en.wikipedia.org/wiki/Cyber-physical_system, 2010.

[3] Taylor T. Johnson, Sayan Mitra, "Handling Failures in CyberPhysical Systems: Potential Directions", Presented at RTSS 2009 PhD Student Forum, December 2009.

[4] S. Bernardi. Building Stochastic Petri Net models for the verification of complex software systems. PhD thesis, Dipartimento di Informatica, Universit a di Torino, April 2003.

[5] LinChuang, Wang Yuan-zhuo, YangYang, QuYang. "Research on network dependability ananlysis methods based on Stochastic Petri net", Journal of electronics, Vol 34, no. 2, 2006, pp.231-332.

[6] E. Lee. Cyber Physical Systems: Design Challenges. University of California, Berkeley Technical Report No. UCB/EECS-2008-8, 2008.

[7] E. Lee. Cyber Physical Systems: Design Challenges. University of California, Berkeley Technical Report No. UCB/EECS-2008-8, 2008.

[8] L. Briesemeister, G. Denker, D. Elenius, I. Mason, S. Varadarajan, D. Bhatt, B. Hall, G. Madl, and W. Steiner. Quantitative fault propagation analysis for networked cyber-physical systems. In 2nd Analytic Virtual Integration of Cyber-Physical Systems Workshop (AVICPS), Nov. 2011.

[9] Chin-Feng Laia, Yi-Wei Maa, Sung-Yen Changa, Han-Chieh Chaob, Yueh-Min Huanga. OSGi-based services architecture for CyberPhysical Home Control Systems, Computer Communications, Vol 34, Issue 2, 2011, pp. 184-191.

[10] D. Harel. Statecharts: A visual formalism for complex systems. Science of Computer Programming, 8: 231-274, 1987.

[11] E. Bode, M. Herbstritt, H. Hermanns, et al. Compositional dependability evaluation for STATEMATE. IEEE Transactions on Software Engineering, 35(2): 274-292, 2009.

[12] T. Murata. Petri nets: properties, analysis and applications. Proceedings of the IEEE, 77(4): 541-580, 1989.

[13] E. Kindler and L. Petrucci. Towards a standard for modular Petri nets: A formalization. Proceedings of the 30th International Conference on Application and Theory of Petri Nets, LNCS 5606, Springer-Verlag, pp. 43-62, 2009.

[14] Z. Manna, A. Pnueli. Temporal Verifications of Reactive Systems Safety, Springer-Verlag, 1995.

[15] E. Vicario, L. Sassoli, and L. Carnevali. Using stochastic state classses in quantitative evaluation of dense-time reactive systems. IEEE Transactions on Software Engineering, 35(5): 703-719, 2009.

[16] M. C. Bujorianu, M. L. Bujorianu, H. Barringer. A formal framework for user centric control of probabilistic multi-agent cyberphysical systems. In M Fisher, et al (Eds.), Computational Logic in Multi-Agent Systems, LNCS 5405, Springer-Verlag, pp.97-116, 2009.

[17] L. Sha, "Using simplicity to control complexity," IEEE Software, vol. 18 , no. 4 , pp. $20-28,2001$.

[18] L. Sha, S. Gopalakrishnan, X. Liu, and Q. Wang. "Cyberphysical systems: A new frontier," in Sensor Networks, Ubiquitous and Trustworthy Computing, 2008. SUTC '08. IEEE International Conference on, June 2008, pp. 1-9.

[19] Chin-Feng Laia, Yi-Wei Maa, Sung-Yen Changa, Han-Chieh Chaob, Yueh-Min Huanga. OSGi-based services architecture for CyberPhysical Home Control Systems, Computer Communications, Vol 34, Issue 2, 2011, pp.184-191.

[20] Liu, Shixi, Hu Xiaojing, and Jingming Wang. "Hierarchical Modeling Fault-Error-Failure Dependencies for Cyber-Physical Systems." Proceedings of The Eighth International Conference on BioInspired Computing: Theories and Applications (BIC-TA), 2013 Springer Berlin Heidelberg, 2013. 